\title{
Isotropic Epsilon-Negative Particles
}

\author{
Jan Machac, Pavel Protiva, Jan Zehentner \\ Czech Technical University in Prague, Technicka 2, 16627 Prague, Czech Republic
}

\begin{abstract}
This paper presents two particles with negative permittivity in some frequency band. The response of a single dipole loaded by an inductance is strongly anisotropic. The response of a spider dipole does not depend on the orientation of the electric field of a wave being incident perpendicular to the particle substrate. These two particles are used in designing an isotropic epsilon-negative metamaterial. A unit cell of the metamaterial consists of a cube bearing the proposed particles on its faces, located with specific orientations. The experiments showed that only the single inductively loaded dipole is suitable for manufacturing of an isotropic epsilon-negative metamaterial. The isotropy of the new particles was checked by measurements.

Index Terms - ENG particle, isotropic metamaterial, negative permittivity.
\end{abstract}

\section{INTRODUCTION}

Metamaterials have become a topic of research in many laboratories. These composite artificial materials offer properties not found in nature, in particular negative permittivity and permeability. Research has focused on materials with both negative permittivity and negative permeability. The first artificial material with negative permittivity was proposed as a $2 \mathrm{D}$ or $3 \mathrm{D}$ lattice of thin wires [1], [2]. Unfortunately, a 3D wire structure is not suitable for mass production. In the first metamaterial [3], a set of parallel wires provided negative permittivity, while split-ring resonators (SRR) produced negative permeability. However, this structure is also not suitable for fabrication. A genuine 3D volumetric metamaterial must be composed of particles known as artificial molecules [4]. There are basically two kinds of such artificial molecules. An electric dipole loaded by an appropriate impedance modifies the permittivity of the host medium by additional polarization due to the induced charge. The planar form of such an epsilon-negative (ENG) particle was presented in [5]. On the other hand, a loop terminated by an appropriate impedance produces an additional magnetization due to the induced current, and changes the permeability of the host medium. SRR is a suitable particle for this purpose.

The basic problem of most metamaterials proposed up to now is their strong anisotropy. Isotropy can be assured by the specific form of a cell and by arranging the particles within this cell in selected crystallographic groups of symmetry. An isotropic mu-negative (MNG) metamaterial was presented in [6] as a uniform 3D structure of cells of cubes with split-ring resonators appropriately located on their faces.

This paper deals with evolution of isotropic ENG metamaterials, and presents two forms of an epsilon-negative particle. The particles are composed of a planar electric dipole loaded by an inductance. The interaction of the exciting field with the particle results in negative permittivity in a narrow band above its resonant frequency. The layouts of these particles are shown in Fig. 1. The single dipole, Fig. 1a, is sensitive only to the electric field parallel to its arms. The spider dipole, Fig. 1b, is insensitive to the electric field orientation provided that the field is parallel to the substrate. The six particles of one kind deposited on their dielectric substrates are assembled to form a cube, as described in [6], to obtain an epsilon-negative cell with an isotropic response. The findings presented in [6] are based on the dipole-to-dipole interactions and higher multipoles are neglected. This is probably not true in the case of the spider dipole, which is not suitable for designing a real volumetric isotropic metamaterial.
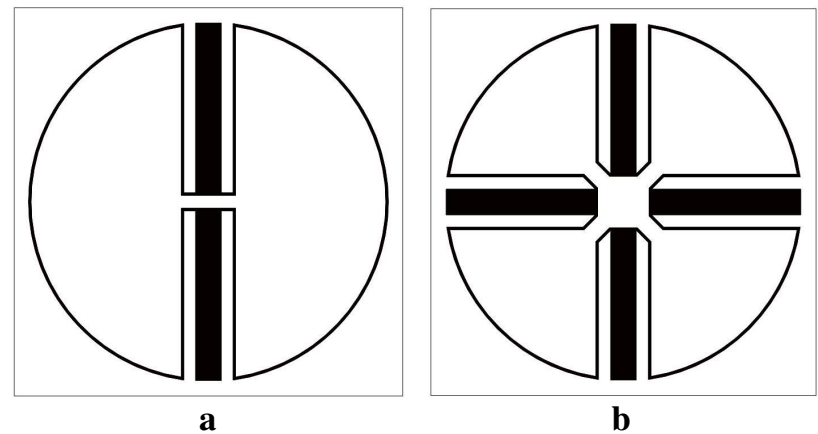

Fig. 1 Layout of epsilon-negative particles, a single electric dipole (a), a spider dipole (b). The rear substrate side is without metallization.

The particles themselves and the cubic cells were designed by the CST Microwave Studio, then fabricated and measured in the rectangular waveguide with the propagating $\mathrm{TE}_{10}$ mode, and in the TEM mode waveguide. The effective permittivity and permeability of the particles were calculated according to [7], although these quantities cannot be defined for a single particle. Our code provides the effective parameters of the volume between the ports where the particle is located, thus they serve as some measure of the electric and magnetic polarizability of the particle. The concept of effective permittivity and permeability will now be used in this sense.

\section{Planar Epsilon-Negative Particles}

Let us see how we can modify the permittivity of a medium. Using a uniform rectangular mesh, let us divide a space into particular cells in the shape of a prism and locate the epsilonnegative particle in the middle of each cell. The dimensions of the cell must be considerably shorter than the wavelength, in 
order to treat the medium as continuous. We will first calculate the electric moment of the dipole. This quantity determines the vector of polarization, and then the effective permittivity of the medium is obtained.

Assuming an electric current $I$ flowing along the arms of the electric dipole, its moment $p$ can be expressed as [8]

$$
p=\frac{I l_{\text {eff }} \cos \theta}{j \omega},
$$

where $l_{\text {eff }}$ is the effective length of the dipole and $\theta$ is the angle between the dipole arms and the electric field vector. Using Ohm's law we get

$$
p=\frac{E l_{\text {eff }}^{2} \cos \theta}{j \omega\left(Z_{e}+Z_{L}\right)},
$$

where $Z_{e}$ and $Z_{L}$ are the dipole input and the loading impedances, respectively, and $E$ is the incident electric field. The magnitude of the polarization vector is

$$
P=\frac{p}{V}=\frac{1}{V} \frac{E l_{e f f}^{2} \cos \theta}{j \omega\left(Z_{e}+Z_{L}\right)}=\alpha_{e} E,
$$

where $V$ is the cell volume, and $\alpha_{e}$ is the electric polarizability, which determines the permittivity of the medium

$$
\varepsilon_{e f f}=1+\frac{\alpha_{e}}{\varepsilon_{0}}=1+\frac{1}{V \varepsilon_{0}} \frac{l_{e f f}^{2} \cos \theta}{j \omega\left(Z_{e}+Z_{L}\right)} .
$$

Loading the electric dipole by an inductor $L_{L}$ at its input terminals and assuming an input dipole capacitance $C_{e}$ we obtain the effective permittivity

$$
\varepsilon_{e f f}=1+\frac{l_{e f f}^{2}}{V \varepsilon_{0}} \frac{C_{e} \cos \theta}{1-\omega^{2} C_{e} L_{L}} .
$$

This represents the classical Lorentz model of a dielectric [8]. The particles presented here do not meet the requirement of being much smaller than the wavelength $(\approx \lambda / 6)$. Consequently, (5) serves only for a qualitative description of the particle response.

We proposed two kinds of ENG particles, see Fig. 1. The single dipole, Fig. 1a, itself represents a particle sensitive only to the electric field parallel to a dipole with an anisotropic response, according to (1). The induced electric dipole moment has its maximum when the electric field is parallel to the dipole arms.

The dipole was fabricated on a ROGERS RT/duroid 5870 substrate with permittivity 2.33 and thickness $0.508 \mathrm{~mm}, \tan \delta$ $=0.0012$ at $10 \mathrm{GHz}$ and metallization thickness $0.035 \mathrm{~mm}$. The dipole, of arm length $13.8 \mathrm{~mm}$ and width $2 \mathrm{~mm}$, is deposited on a substrate area $30 \times 30 \mathrm{~mm}$. The measured and calculated transmissions of this dipole are plotted in Fig. 2. The dipole was located in the R18 waveguide at the center of its cross-section. The CST Microwave Studio uses a rectangular mesh and therefore is not able precisely to discretize the circularly bent strips of the inductance. This causes the difference between the measured and simulated patterns, as shown in Fig. 2. However, with increasing mesh density limited by computer memory, and by computation time, the simulated pattern in Fig. 2 approaches the measured pattern. The measurement was done at various angles $\theta$, specified in Fig. 2, from which the anisotropic particle behavior is seen, as anticipated above. With increasing angle, the particle response decreases in proportion to $\cos \theta$, and the resonant frequency is shifted down. Finally there is zero response for $\theta=90^{\circ}$. Fig. 3 shows the calculated real and imaginary parts of the complex effective permittivity provided by this dipole occupying its basic position when $\theta=0^{\circ}$.

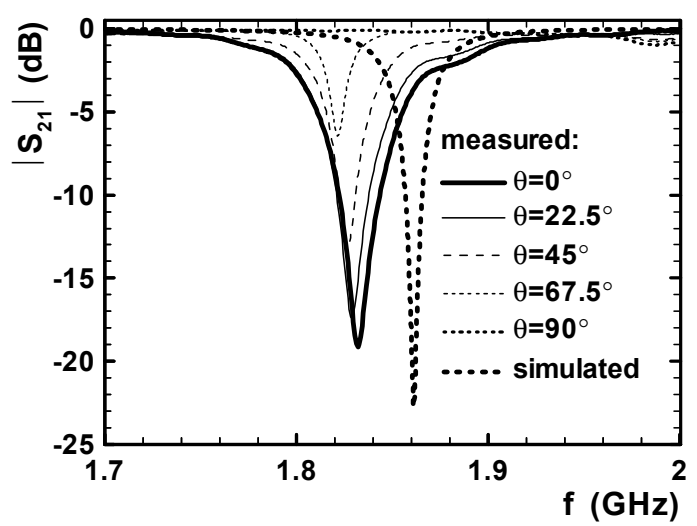

Fig. 2 Transmission of the single dipole from Fig. 1a.

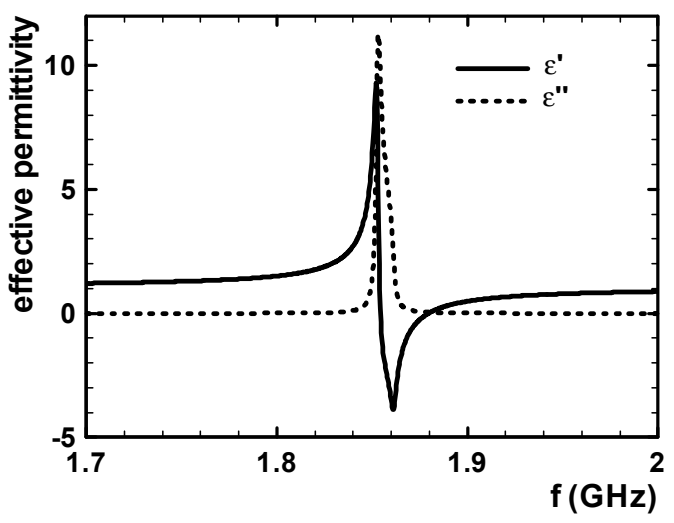

Fig. 3 Calculated effective permittivity of the single dipole from Fig. 1a corresponding to the simulated transmission in Fig. 2.

A spider dipole was fabricated using the same substrate and similar proportions as in the case of the single dipole. Due to its symmetry, this particle has an isotropic response, assuming that the wave is incident perpendicular to the substrate. This is documented in Fig. 4, where the measured transmissions of 
this dipole are plotted for three different angles $\theta$ between the electric field and the dipole arms. The measurement was taken in the R18 waveguide. The responses in these three cases are almost identical.

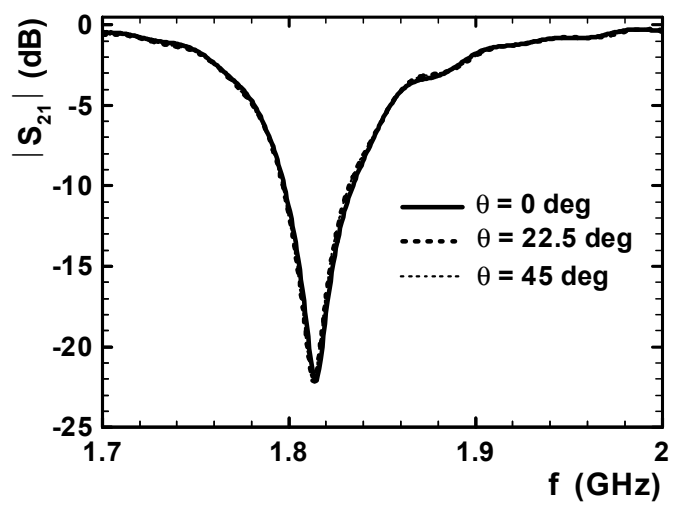

Fig. 4 Measured transmissions of the spider dipole in Fig. 1b for three positions of its declinations from the vertical position.

\section{Volumetric EPSILON-Negative PARTiCle}

The 2D planar particles introduced in Section II were used for designing the 3D isotropic unit cell, which provides negative permittivity. This idea can be carried into effect by placing the planar particles on the faces of a cube, observing the appropriate crystallographic groups of symmetry [6]. The particles from Fig. 1a located in the positions on the faces of the cube create a tetrahedral symmetrical system [6], see Fig. $5 \mathrm{a}$, while the particles from Fig. $1 \mathrm{~b}$ positioned according to Fig. $5 \mathrm{~b}$ provide an octahedral symmetrical system [6]. The interior of the cube is empty, since the particle substrates themselves create the faces of the cube. Fig. 5 shows the basic orientation of the cubes, assuming that the incident electric field is directed parallel to the $y$ axis.

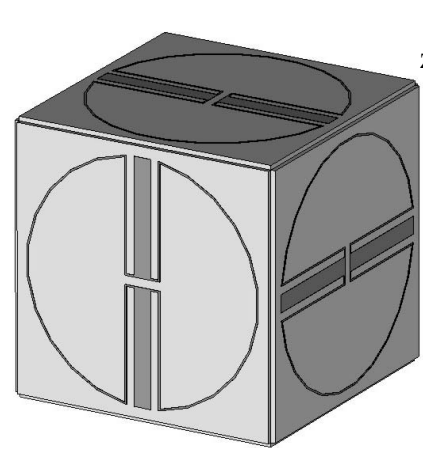

(a)

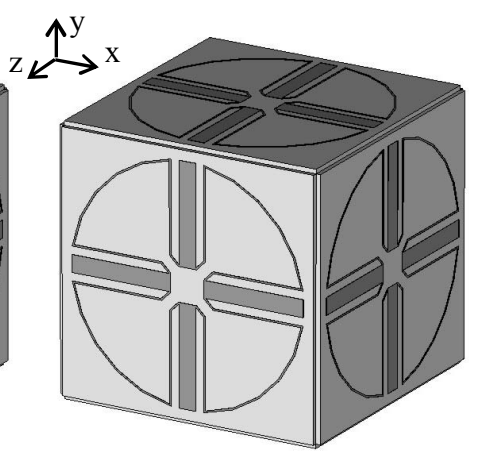

(b)
Fig. 5 Volumetric epsilon-negative particles composed of single dipoles (a), and composed of spider dipoles (b).

The cubes composed of particular planar particles are complex systems with many internal couplings. These structures were studied in the R18 waveguide and also in the waveguide supporting the TEM wave. The presence of waveguide walls, the existence of the $\mathrm{TE}_{10}$ mode longitudinal magnetic field component and the non-homogeneity of this field influence the electromagnetic response of the particle inserted in the waveguide. These effects result in different responses of the cubes in comparison with the responses of the single planar particles.

The measured transmissions of the cube composed of spider dipoles, Fig. 5b, in the R18 waveguide taken for selected orientations, are plotted in Fig. 6. As to notation, figure 1 means the basic orientation, $8_{i}$ represents the rotation around the $i$ axis by angle $2 \pi / 8$, i.e., $45^{\circ}$, where $i=x, y, z$. The measurements of this particle performed in the TEM waveguide provided similar responses. Consequently, this cube is not isotropic at all, as its response depends on the orientation. This is in contradiction with the conclusion in [6] that the octahedral symmetry of this cube should provide an isotropic response. The conclusions in [6] are based on dipoleto-dipole interaction of particles and on neglecting higher order multipoles. It can be assumed that in a cube of spider dipoles the effect of higher order multipoles is not negligible.

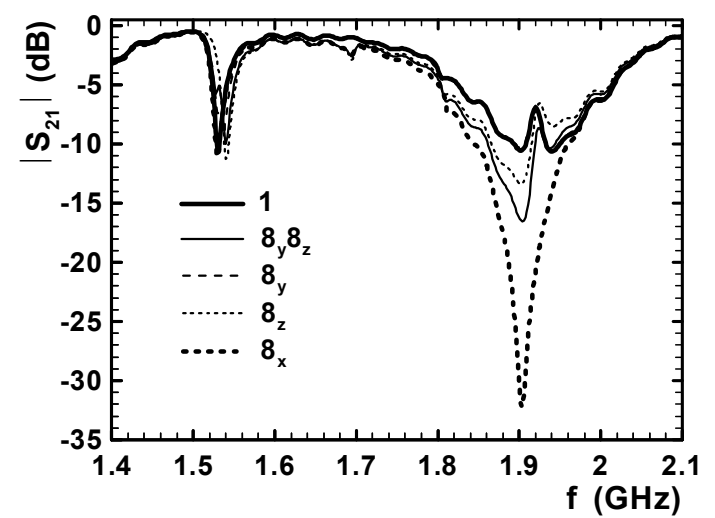

Fig. 6 Measured transmissions of a cube of spider dipoles for its different orientations in the R18 waveguide.

Fig. 7 shows the transmissions of the cube assembled of single dipoles from Fig. 5a, inserted into the R18 waveguide and measured for several orientations. Now $4_{y}$ represents the rotation around the $y$ axis by $2 \pi / 4$, i.e., $90^{\circ}$. The transmissions of this cube calculated by the CST Microwave Studio differ from the measured patterns similarly as in Fig. 2. The transmissions of this cube measured in the TEM waveguide are plotted in Fig. 8 for the same orientations selected in Fig. 7. The cube response around the resonance at $1.82 \mathrm{GHz}$, as shown in Figs. 7 and 8, is almost insensitive to the particle orientation: consequently, this cube is isotropic. The calculated real part of the complex effective permittivity $\mathcal{\varepsilon}$ and permeability $\mu$ ' of this cube for its basic orientation is depicted in Fig. 9. The two resonances shown in Fig. 9 correspond to the two resonances shown in Fig. 7. Tightly above the higher resonance, the cube behaves as an epsilonnegative particle. The response round the lower resonance depends slightly on the orientation of the cube: therefore the cube is not an isotropic mu-negative particle. 


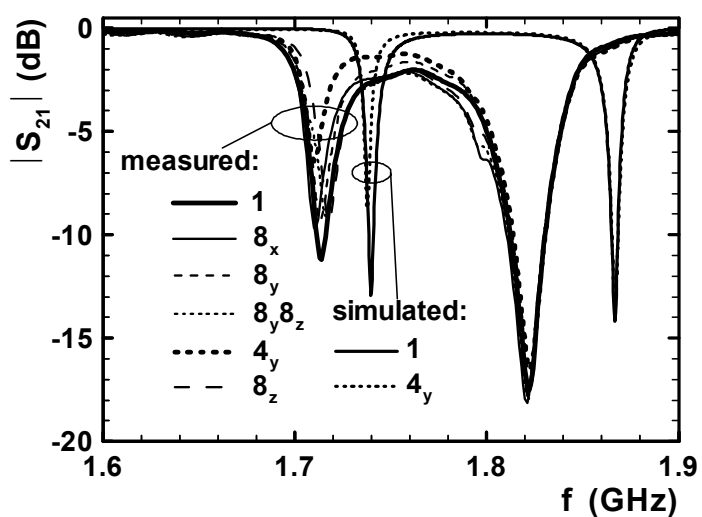

Fig. 7 Transmissions of the cube assembled of single dipoles from Fig. 5a for its different orientations in the R18 waveguide.

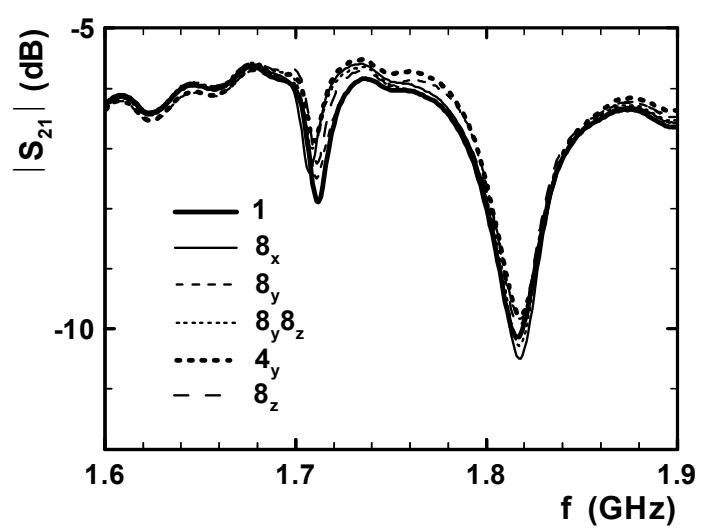

Fig. 8 Measured transmissions of the cube assembled of single dipoles from Fig. 5a for its different orientations in the TEM waveguide.

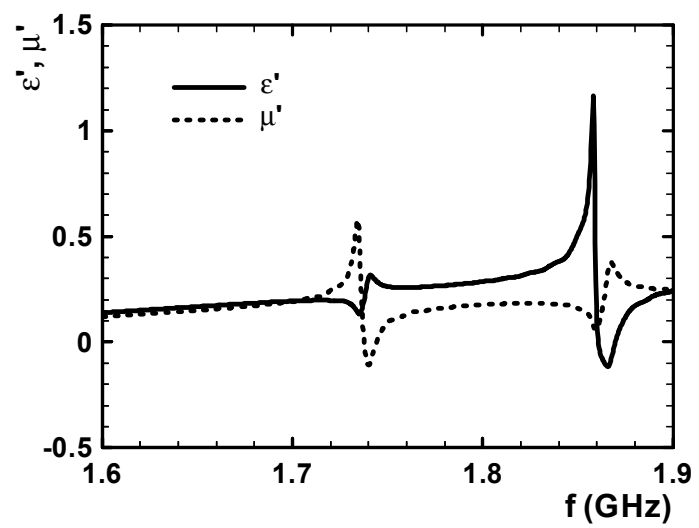

Fig. 9 The calculated effective parameters of the cube assembled of single dipoles from Fig. 5a correspond to the simulated transmission characteristic in Fig. 7.

\section{CONCLUSIONS}

Two epsilon-negative particles are presented in this paper. A single dipole consists of a planar dipole loaded by a loop representing an inductor. This particle response is anisotropic. A spider dipole consists of a couple of dipoles located perpendicular to each other on a substrate and connected by loop inductors. The response of this epsilon-negative particle is insensitive to the orientation of the electric field of the wave incident perpendicular to the substrate. These dipoles were tested for the assembly of an isotropic epsilon-negative metamaterial. They served as the faces of a cube and fulfilled constraints ensuring the required crystallographic symmetries. At higher resonance, a cube consisting of single dipole particles has an isotropic response with negative permittivity, while at lower resonance it exhibits a mu-negative response. The cube composed of the spider dipoles did not show the expected isotropic epsilon-negative response.

\section{ACKNOWLEDGEMENT}

This work has been supported by the Grant Agency of the Czech Republic under project 102/06/1106 "Metamaterials, nanostructures and their applications".

\section{REFERENCES}

[1] J. B. Pendry, A. J. Holden, W. J. Stewart and I. Youngs, "Extremely Low Frequency Plasmons in Metallic Mesostructures," Phys. Rev. Lett, vol 76, no. 25, pp. 4773-4776, June 1996.

[2] J. B. Pendry, A. J. Holden, D. J. Robbins and W. J. Stewart, "Low Frequency plasmons in Thin-Wire Structures," Journal of Physics: Condensed Matter, vol. 10, pp. 4785-4809, 1998.

[3] D. R. Smith, W. J. Padilla, D. C. Vier, S. C. Nemat-Nasser and S. Schultz, "Composite Medium with Simultaneously Negative Permeability and Permittivity," Phys. Rev. Lett., vol. 84, no. 18, pp.4184-4187, May 2000.

[4] R. W. Ziolkowski and F. Auzanneau, "Passive artificial molecule realizations of dielectric materials," J. Appl. Phys, vol. 82, no. 7, pp. 3195-3198, October 1997.

[5] D. Schuring, J. J. Mock and D. R. Smith, "Electric-FieldCoupled Resonators for Negative Permittivity Metamaterials," Applied Phys. Lett, vol. 88, 041109, 2006.

[6] J. D. Baena, L. Jelinek, R. Marques and J. Zehentner, "Electrically Small Isotropic Three-Dimensional Magnetic Resonators for Metamaterial Design”, Applied Phys. Lett, vol. 88, 134108, 2006.

[7] D. R. Smith, S. Schultz, P. Markos, C. S. Soukoulis: Determination of effective permittivity and permeability of metamaterial from reflection and transmission coefficients, Phys. Rev. B, vol 65, 195104, 2002.

[8] F. Auzanneau, R. W. Ziolkowski: Theoretical study of synthetic bianisotropic materials, J. of Electromagnetic Waves and Applications, vol. 12, 1998, pp. 353-370. 